Modified Laplace-Beltrami quantization of natural Hamiltonian systems with quadratic constants of motion

Claudia Maria Chanu, Luca Degiovanni, and Giovanni Rastelli

Citation: Journal of Mathematical Physics 58, 033509 (2017); doi: 10.1063/1.4978326

View online: http://dx.doi.org/10.1063/1.4978326

View Table of Contents: http://aip.scitation.org/toc/jmp/58/3

Published by the American Institute of Physics

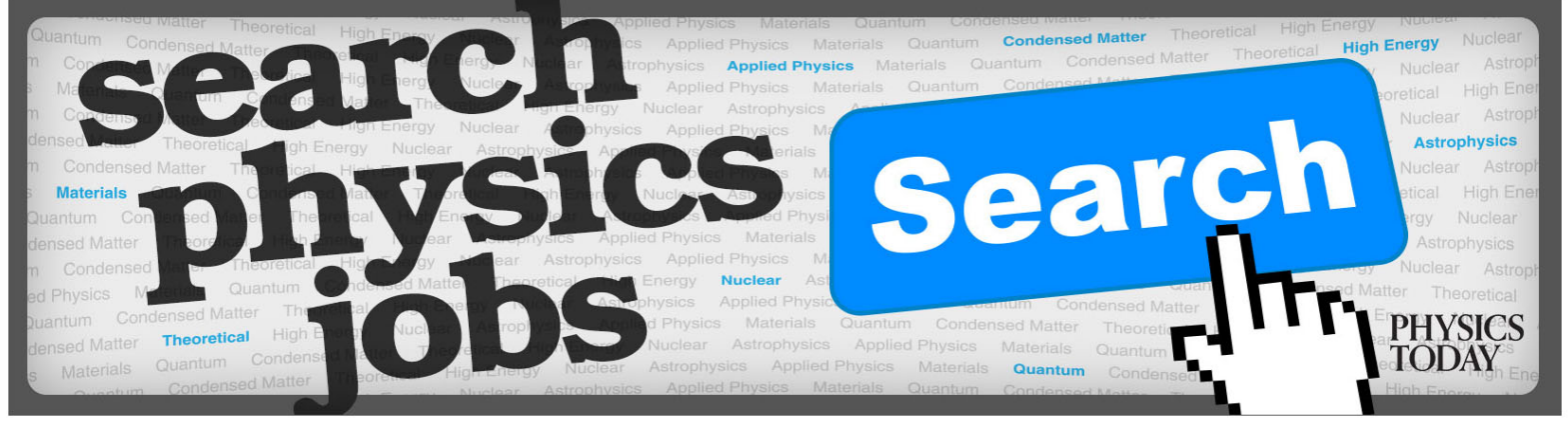




\title{
Modified Laplace-Beltrami quantization of natural Hamiltonian systems with quadratic constants of motion
}

\author{
Claudia Maria Chanu, ${ }^{\text {a) }}$ Luca Degiovanni, ${ }^{\text {b) }}$ and Giovanni Rastellic) \\ Dipartimento di Matematica, Università di Torino, via Carlo Alberto 10, Torino, Italy
}

(Received 3 October 2016; accepted 25 February 2017; published online 30 March 2017)

\begin{abstract}
It is natural to investigate if the quantization of integrable or superintegrable classical Hamiltonian systems is still integrable or superintegrable. We study here this problem in the case of natural Hamiltonians with constants of motion quadratic in the momenta. The procedure of quantization here considered transforms the Hamiltonian into the Laplace-Beltrami operator plus a scalar potential. In order to transform the constants of motion into symmetry operators of the quantum Hamiltonian, additional scalar potentials, known as quantum corrections, must be introduced, depending on the Riemannian structure of the manifold. We give here a complete geometric characterization of the quantum corrections necessary for the case considered. In particular, Stäckel systems are studied in detail. Examples in conformally and non-conformally flat manifolds are given. Published by AIP Publishing. [http://dx.doi.org/10.1063/1.4978326]
\end{abstract}

\section{INTRODUCTION}

When a classical natural Hamiltonian $H$ defined on the cotangent bundle of a Riemannian manifold $M$ admits a quadratic in the momenta first integral $K$, the construction of pairwise commuting second order differential operators associated with $H$ and $K$ is a non-trivial problem. One of the possible quantization rules is the so-called Laplace-Beltrami quantization (or minimal quantization, or Carter quantization): if we consider the cotangent bundle $T^{*} M$ of a $N$-dimensional Riemannian manifold $(M, \mathbf{g})$, then the Laplace-Beltrami (LB) quantization associates with each quadratic in the momenta function (without terms linear in the momenta)

$$
K=\frac{1}{2} K^{a b} p_{a} p_{b}+W
$$

defined by the symmetric two-tensor $\mathbf{K}$ and the scalar $W$, the second-order differential operator on functions on $M$,

$$
\widehat{K}=-\frac{\hbar^{2}}{2} \Delta_{K}+W=-\frac{\hbar^{2}}{2} \nabla_{a} K^{a b} \nabla_{b}+W
$$

where $\nabla_{a}$ is the covariant derivative with respect to the Levi-Civita connection of the metric $\mathbf{g}$. In particular, the operator associated with a geodesic Hamiltonian is (up to the constant factor $-\hbar^{2} / 2$ ) the Laplace-Beltrami operator $\Delta$.

When the configuration manifold $M$ is conformally flat, other ways of matching quadratic first integrals of natural Hamiltonians (including $H$ itself) with differential operators on a Hilbert space have been employed, in order to obtain integrability or superintegrability of the quantum versions of specific Hamiltonian systems, see, for example, the articles. ${ }^{1,15}$ Essentially, in both papers the quantization procedure requires a "quantum correction" of the Laplacian: an additional scalar term proportional to the scalar curvature Sc appears in the quantum Hamiltonian and in its symmetry

\footnotetext{
a)e-mail: claudiamaria.chanu@unito.it

b) e-mail: luca.degiovanni@gmail.com

c)e-mail: giovanni.rastelli@unito.it
} 
operators. The quantum corrections are necessary in order to allow the commutation with the Hamiltonian operator of one or more of the quantized constants of motion.

Namely, in Ref. 1 (beside other two coordinate dependent techniques of quantization), it is shown that the maximal superintegrability of a classical $N$-dimensional Hamiltonian system is kept after quantization only if to the natural Hamiltonian

$$
H=\frac{1}{2} g^{a b} p_{a} p_{b}+V
$$

is associated with the symmetry operator

$$
\widehat{H}_{c}=-\frac{\hbar^{2}}{2}\left(\Delta-\frac{N-2}{4(N-1)} \mathrm{Sc}\right)+V,
$$

where $\Delta$ is the Laplace-Beltrami operator and $\mathrm{Sc}$ is the scalar curvature of the metric $\mathrm{g}$. The operator $\Delta_{c}$,

$$
\Delta_{c}=\Delta-\frac{N-2}{4(N-1)} \mathrm{Sc},
$$

is the well known conformally invariant Laplacian (also known as Yamabe operator). A specific study on symmetries and conformal symmetries of the conformally invariant Laplacian is given in Ref. 17, where the quantization procedure considered is the so-called "conformally equivariant quantization" developed in Refs. 13 and 14. In this type of quantization, equivariance with respect to the action of the conformal symmetry group is required, and the classical configuration manifolds are assumed to be conformally flat. In Ref. 17, the hypothesis of conformal flatness is dropped and a geometrical compatibility condition is given, relating the (conformal) Killing tensor $\mathbf{K}$, the Cotton-York tensor $\mathbf{A}$, and the Weyl tensor $\mathbf{C}$. If the condition is satisfied, then a constructive method allows us to write a (conformal) symmetry of the conformally invariant Laplacian (1). Moreover, all (conformal) Killing tensors defined on a conformally flat manifold satisfy this condition, and this explains the quantum corrections proposed in Ref. 1.

In the present paper, instead, we look for a pair of additional functions $E$ and $E_{K}$ that have to be added to the Laplacian and to the Laplace-Beltrami quantization $\Delta_{K}$ of a quadratic in the momenta first integral $K$ in order to get two commuting differential operators. These functions, called quantum corrections, are in principle both considered as unknown to be determined. The quantization procedure obtained in this way will be called modified Laplace-Beltrami (MLB) quantization.

A compatibility condition involving both $E$ and $E_{K}$ is established and a consequent integrability condition involving only $E$ is derived. If we assume that $E$ is the scalar curvature, then this integrability condition reduces to the one proposed in Ref. 17. In this case, the modified Hamiltonian quantum operator coincides with the conformally invariant Laplacian (1). By leaving $E$ arbitrary, instead, we have the possibility to find suitable quantum corrections for non-conformally flat metrics also (see, for example, Refs. 17 and 16 in four dimension). For instance, a more geometric interpretation is proposed for the quantum correction given in Ref. 16 and we explicitly write commuting operators up to the five dimensional case.

Our analysis is carried on for any quadratic constants of motion of any natural Hamiltonian, and we obtain necessary and sufficient conditions for the existence of quantum corrections for the simultaneous quantization of all the quadratic constants of motion of the classical system.

In Section II, we introduce the Laplace-Beltrami quantization and we obtain our main results: Theorems 1,5 , and 6 .

Through Theorem 1, we characterize the quantum corrections of the Laplace-Beltrami operator and of its second-order symmetry operator involving the Ricci tensor, the Killing two-tensor associated with the quadratic first integral, and the Levi-Civita connection.

Theorem 5 characterizes the quantum corrections necessary to the simultaneous modified quantization of several quadratics in the momenta first integrals of the same Hamiltonian.

In Theorem 6, we restrict the previous results to the important case of Stäckel systems, the $N$-dimensional Hamiltonian systems admitting $N-1$ quadratic in the momenta first integrals in involution, plus the Hamiltonian itself, associated with the existence of coordinate systems allowing the integration by separation of variables of the Hamilton-Jacobi equation. The Laplace-Beltrami 
quantization of these systems has been considered in Refs. 2 and 3 and we recall the main results. We address here to the case when the LB operator has to be modified by some quantum correction, for example, due to the existence of additional quadratic constants of motion not included into the involutive set, a situation typical of superintegrable systems. We give necessary conditions for the quantum correction which allows the simultaneous modified quantization of the whole Stäckel system.

In Section III, we apply the results of Section II to three examples. The first two, taken from Ref. 17, show that the initial freedom in the choice of the quantum correction allows the quantization of first integrals found to be not associated with symmetries of the conformally invariant Laplacian in Ref. 17. In the third example, inspired by Ref. 16, we apply our technique to a hierarchy of $\mathrm{N}$ dimensional Hamiltonian systems, all maximally superintegrable with $2 N-1$ constants of motion all quadratics in the momenta, defined (for $N>4$ ) on non-conformally flat manifolds. We discuss the geometric meaning of some of the quantum corrections considered in Ref. 16.

\section{MODIFIED LAPLACE-BELTRAMI QUANTIZATIONS}

In order to study a modified Laplace-Beltrami (MLB) quantization, we generalize here some results obtained in Ref. 3 about the Laplace-Beltrami quantization. In Ref. 3, the Laplace-Beltrami quantization of quadratic functions on cotangent bundles of Riemannian and pseudo-Riemannian manifolds was considered. Theorem 2.2 states that, given the quadratic in the momenta functions

$$
H=\frac{1}{2} g^{a b} p_{a} p_{b}+W, \quad K=\frac{1}{2} K^{a b} p_{a} p_{b}+W_{K},
$$

and the operators

$$
\widehat{H}=-\frac{\hbar^{2}}{2} \Delta+W, \quad \widehat{K}=-\frac{\hbar^{2}}{2} \Delta_{K}+W_{K}
$$

with

$$
\Delta_{K}=\nabla_{a} K^{a b} \nabla_{b},
$$

then $[\widehat{H}, \widehat{K}]=0$ (i.e., $\widehat{H}$ and $\widehat{K}$ commute) if and only if the 2-tensor $\mathbf{K}$ of contravariant components $\left(K^{a b}\right)$ is a Killing tensor for the metric $\mathbf{g}$ and

$$
\mathbf{K} \nabla W-\nabla W_{K}+\frac{\hbar^{2}}{6} \delta \mathbf{C}_{K}=0,
$$

where

$$
\mathbf{C}_{K}=\mathbf{K R}-\mathbf{R K},
$$

$\mathbf{R}$ is the Ricci tensor, and $\left(\delta C_{K}\right)^{a}=\nabla_{b} C_{K}^{b a}$ (i.e., $\delta$ is the divergence operator). It is well known that $\{H, K\}=0$ ( $H$ and $K$ Poisson-commute) if and only if $\mathbf{K}$ is a Killing tensor and $\mathbf{K} \nabla W-\nabla W_{K}=0$, therefore, Proposition 2.5 of Ref. 3 states that, for any quadratic first integral $K$ of $H$,

$$
[\widehat{H}, \widehat{K}]=0 \Leftrightarrow \delta \mathbf{C}_{K}=0 .
$$

Definition 1. We say that $\mathbf{K}$ satisfies the Carter condition if $\delta \mathbf{C}_{K}=0$.

Remark 1. The Laplace-Beltrami quantization (2) provides (formally) self-adjoint differential operators acting on functions (see Ref. 3). The modification of the Laplacian by an additional scalar term does not affect self-adjointness.

Remark 2. If we are dealing with a constant-curvature configuration manifold or, more generally, with any Einstein manifold, where the Ricci tensor is a multiple of the metric, then the LaplaceBeltrami quantization produces symmetry operators for the quantum Hamiltonian operator for each quadratic first integral of the classical Hamiltonian.

Remark 3. If the Killing tensor $\mathbf{K}$ and the Ricci tensor $\mathbf{R}$ are simultaneously diagonalized in some coordinate system, then the Carter condition is automatically satisfied, because $\mathbf{C}_{K}=0$. 
We adapt the previous results to the case of the modified quantization. We have the following.

Theorem 1. Let $\Delta_{E}$ be the modified Laplacian $\Delta_{E}=\Delta+E$, with $E$ any given scalar, $\widehat{H}_{E}$ $=-\frac{\hbar^{2}}{2} \Delta_{E}+V=-\frac{\hbar^{2}}{2}(\Delta+E)+V$, and $\widehat{K}_{E_{K}}=-\frac{\hbar^{2}}{2}\left(\Delta_{K}+E_{K}\right)+V_{K}$, where $E_{K}$ is a scalar to be determined, then

$$
\left[\widehat{H}_{E}, \widehat{K}_{E_{K}}\right]=0
$$

if and only if

(i) the 2-tensor $\mathbf{K}$ of contravariant component $\left(K^{a b}\right)$ is a Killing tensor;

(ii) the following equation holds:

$$
\frac{\hbar^{2}}{6} \delta \mathbf{C}_{K}+\frac{\hbar^{2}}{2}\left(\nabla E_{K}-\mathbf{K} \nabla E\right)+\mathbf{K} \nabla V-\nabla V_{K}=0 .
$$

Proof. The modified operators $\widehat{H}_{E}$ and $\widehat{H}_{K_{E}}$ correspond to the Laplace-Beltrami quantization (2) of the Hamiltonians $H_{E}=\frac{1}{2} g^{a b} p_{a} p_{b}+W, K_{E_{K}}=\frac{1}{2} K^{a b} p_{a} p_{b}+W_{K}$, where

$$
\begin{gathered}
W=V-\frac{\hbar^{2}}{2} E, \\
W_{K}=V_{K}-\frac{\hbar^{2}}{2} E_{K},
\end{gathered}
$$

and we may apply Theorem 2.2 of Ref. 3. By substituting these relations in (3), we obtain

$$
\mathbf{K}\left(\nabla V-\frac{\hbar^{2}}{2} \nabla E\right)-\nabla V_{K}+\frac{\hbar^{2}}{2} \nabla E_{K}+\frac{\hbar^{2}}{6} \delta \mathbf{C}_{K}=0
$$

that gives Equation (4).

Since $H_{K}$ is a first integral of $H$ if and only if $\mathbf{K}$ is a Killing tensor and

$$
\mathbf{K} \nabla V-\nabla V_{K}=0,
$$

it follows that.

Proposition 2. If $\{H, K\}=0$, then $\left[\widehat{H}_{E}, \widehat{K}_{E_{K}}\right]=0$ if and only if

$$
\nabla E_{K}-\mathbf{K} \nabla E+\frac{1}{3} \delta \mathbf{C}_{K}=0 .
$$

From Proposition 2, integrability conditions for $E_{K}$ can be immediately obtained.

Proposition 3. The integrability conditions of $E_{K}$ coincide with the symmetry of the 2-tensor $\mathbf{P}=\nabla\left(\mathbf{K} \nabla E-\frac{1}{3} \delta \mathbf{C}_{K}\right)$ of covariant components

$$
P_{a b}=\nabla_{a}\left[(K \nabla E)_{b}-\frac{1}{3}\left(\delta C_{K}\right)_{b}\right] .
$$

Equivalently,

$$
d\left(\mathbf{K} \nabla E-\frac{1}{3} \delta \mathbf{C}_{K}\right)^{b}=0 .
$$

Proposition 4. Let $\left(E, E_{K}\right)$ be a pair of functions satisfying condition (5). The most general solutions $\left(E_{g}, E_{K g}\right)$ of (5) are of the form

$$
E_{g}=E+E_{o}, \quad E_{K g}=E_{K}+E_{K o},
$$

with $\left(E_{o}, E_{K o}\right)$ belonging to the linear space defined by the solutions of the linear homogeneous differential equation

$$
\mathbf{K} \nabla E_{o}=\nabla E_{K o}
$$

whose integrability condition is $d\left(\mathbf{K} \nabla E_{o}\right)^{b}=0$.

Proof. Equation (5) is a linear non-homogeneous differential condition in $E$ and $E_{K}$, whose associated homogeneous equation is (7). 
Condition (7) is equivalent to $\left\{H+E_{o}, K+E_{K o}\right\}=0$.

When a Hamiltonian $H$ admits several quadratic first integrals and we know the quantum corrections allowing the MLB quantization of $H$ together with each one of the first integrals, we may ask ourselves if there exists a single quantum correction of $H$ allowing the simultaneous MLB quantization of all those first integrals. We have the following.

Theorem 5. Let $H$ be a Hamiltonian with $k$ quadratic in the momenta first integrals $K^{(i)}$. Let us assume that for each $i$ there exist quantum corrections $E^{(i)}, F^{(i)}$ such that

$$
\left[\widehat{H}_{E^{(i)}}, \widehat{K}_{F^{(i)}}^{(i)}\right]=0 .
$$

There exists a simultaneous quantum correction $E$ of $H$ for all $K^{(i)}$ if and only if

$$
d\left(\mathbf{K}^{(i)} \nabla E^{(i)}\right)=d\left(\mathbf{K}^{(i)} \nabla E\right), \quad i=1, \ldots, k .
$$

Moreover, the quantum corrections of $\mathbf{K}^{(i)}$ corresponding to $E$ are $W^{(i)}=F^{(i)}+\bar{F}^{(i)}$, where each $\bar{F}^{(i)}$ is a potential of the closed one-form $\mathbf{K}^{(i)} \nabla\left(E-E^{(i)}\right)$, then

$$
\left[\widehat{H}_{E}, \widehat{K}_{W^{(i)}}^{(i)}\right]=0 .
$$

Proof. From Proposition 2, a simultaneous quantum correction $E$ exists if and only if it satisfies

$$
\frac{1}{3} \delta \mathbf{C}_{K^{(i)}}=\mathbf{K}^{(i)} \nabla E-\nabla W^{(i)}, \quad i=1, \ldots, k,
$$

for suitable functions $W^{(i)}$. From the same proposition, we have

$$
\frac{1}{3} \delta \mathbf{C}_{K^{(i)}}=\mathbf{K}^{(i)} \nabla E^{(i)}-\nabla F^{(i)}, \quad i=1, \ldots, k,
$$

then we obtain the equations $(i=1, \ldots, k)$

$$
\mathbf{K}^{(i)} \nabla\left(E^{(i)}-E\right)=\nabla\left(F^{(i)}-W^{(i)}\right) .
$$

The integrability condition of these equations are

$$
d\left(\mathbf{K}^{(i)} \nabla\left(E^{(i)}-E\right)\right)=d\left(\mathbf{K}^{(i)} \nabla E^{(i)}\right)-d\left(\mathbf{K}^{(i)} \nabla E\right)=0 .
$$

We remark that the previous statement does not consider the possible commutation relations among the first integrals, required by Liouville and quantum integrability of the Hamiltonian.

Stäckel systems are natural $N$-dimensional Hamiltonian systems with $N$ quadratic independent first integrals in involution, such that the Killing tensors associated with these first integrals are simultaneously diagonalized in orthogonal coordinates, coordinates determined by the eigenvectors of the Killing tensors. These coordinates are called Stäckel coordinates. It follows ${ }^{2}$ that Stäckel systems are Liouville integrable, one of the first integrals can be chosen as the Hamiltonian $H$ itself. The characteristic property of Stäckel systems is that the Hamilton-Jacobi equation of $H$ is additively separable in Stäckel coordinates. We call Killing-Stäckel algebra the linear space generated by the Killing tensors of a Stäckel system.

It is known (Refs. 2 and 3 and the references therein) that the Schrödinger equation is multiplicatively separable in Stäckel coordinates if and only if the coordinates diagonalize the Ricci tensor also. In this case, the condition $C_{K}=0$ is verified for all the elements of the Killing-Stäckel algebra.

Moreover, Stäckel systems are LB quantizable provided the Carter condition holds for the KillingStäckel algebra. We recall that, for a Stäckel system, ${ }^{2}$

$$
\delta \mathbf{C}_{K_{i}}=0, i=1, \ldots, N, \Leftrightarrow\left[\widehat{K}_{i}, \widehat{K}_{j}\right]=0, i, j=1, \ldots, N .
$$


Consequently, in this case no quantum correction is necessary. However, if the Stäckel system is superintegrable, quantum corrections can be necessary to quantize the additional first integrals.

If the Carter condition holds for all the quadratic Killing 2-tensors $\left(\mathbf{K}_{i}\right), i=1, \ldots, N$, of a KillingStäckel algebra, we call it pre-Robertson condition. If, in addition, $\mathbf{C}_{K i}=0, i=1, \ldots, N$, we say that $\left(\mathbf{K}_{i}\right)$ satisfy the Robertson condition (see Refs. 12 and 2 and the references therein).

In Stäckel coordinates, since $\mathbf{K}_{i}$ are simultaneously diagonalized, the Robertson condition is equivalent to the diagonalization of the Ricci tensor. As shown in Ref. 3, the satisfaction of the pre-Robertson condition allows the LB quantization of the Killing-Stäckel algebra in any manifold.

The LB quantization of Stäckel systems, discussed in Refs. 2 and 3, provides a Schrödinger operator that admits the orthogonal (multiplicative) separation of variables in the same orthogonal coordinates allowing the (additive) separation of variables for the Hamilton-Jacobi equation of the original Stäckel system, only if the Robertson condition is satisfied.

In Ref. 5, an alternative quantization procedure of Stäckel systems is considered and applied in Ref. 6 to a class of superintegrable Stäckel systems with all quadratics in the momenta constants of motion. The procedure gives separable, and superintegrable, Schrödinger operators even when the Robertson condition is not verified. This result is obtained by replacing the metric tensor $\mathbf{g}$ defining the Laplace-Beltrami operator with a suitable conformal deformation $\overline{\mathbf{g}}$. An analysis of the similarities and differences between these two approaches is worthwhile, but beyond the scope of the present paper.

From Theorem 1 and Theorem 5, we derive the following result, useful for the quantization of superintegrable Stäckel systems.

Theorem 6. Let $\left(H, K_{1} \ldots, K_{N-1}\right)$ be a Stäckel system, of Hamilton function $H$ and separable coordinates $\left(q^{i}\right)$, satisfying the pre-Robertson condition. Let $\mathbf{K}_{i}$ be the Killing tensors such that $K_{i}=\frac{1}{2} K_{i}^{l m} p_{l} p_{m}+V_{i}$. Let $\widehat{H}_{E}$ be the quantum Hamiltonian obtained from $H$ with any quantum correction E. If

$$
d\left(\mathbf{K}_{i} d E\right)=0, \quad i=1, \ldots, n-1,
$$

then the system $\left(H-\frac{\hbar^{2}}{2} E, K_{1}-\frac{\hbar^{2}}{2} E_{1} \ldots, K_{N-1}-\frac{\hbar^{2}}{2} E_{N-1}\right)$, where $d E_{i}=\mathbf{K}_{i} d E$, is a Stäckel system separable in $\left(q^{i}\right)$. Moreover, by denoting $\widehat{K}_{E_{i}}$ the modified operators with quantum corrections $E_{i}$, the system $\left(\widehat{H}_{E}, \widehat{K}_{E_{1}}, \ldots, \widehat{K}_{E_{N-1}}\right)$ is a quantum integrable system. Furthermore, if the stronger Robertson condition is satisfied by the Stäckel system, then the quantum system $\left(\widehat{H}_{E}, \widehat{K}_{E_{1}}, \ldots, \widehat{K}_{E_{N-1}}\right)$ is also separable in $\left(q^{i}\right)$.

Proof. Since the pre-Robertson condition is satisfied, Equation (5) of Proposition 2 reads as

$$
\nabla E_{K_{i}}=\mathbf{K}_{i} \nabla E=0, \quad i=1, \ldots, N-1,
$$

whose integrability conditions are

$$
d\left(\mathbf{K}_{i} d E\right)=0 .
$$

Since $\mathbf{K}_{i}$ are Killing tensors, Equation (11) means that

$$
\left\{H-\frac{\hbar^{2}}{2} E, K_{i}-\frac{\hbar^{2}}{2} E_{i}\right\}=0,
$$

and, in separable coordinates $\left(q^{j}\right)$ for the Stäckel system $\left(H, K_{1} \ldots, K_{N-1}\right)$, that $E=\phi_{j}\left(q^{j}\right) g^{j j}$, i.e., $E$ is a Stäckel multiplier, ${ }^{2}$ as well as $-\frac{\hbar^{2}}{2} E$. Therefore, also the commutation conditions

$$
\left\{H_{i}-\frac{\hbar^{2}}{2} E_{i}, H_{j}-\frac{\hbar^{2}}{2} E_{j}\right\}=0
$$

follow and $\left(H-\frac{\hbar^{2}}{2} E, K_{1}-\frac{\hbar^{2}}{2} E_{1} \ldots, K_{N-1}-\frac{\hbar^{2}}{2} E_{N-1}\right)$ is again a Stäckel system. Since the Killing tensors $\mathbf{K}_{i}$ and the Ricci tensor are unchanged, the pre-Robertson condition still holds true for the new system and the separable coordinates are the same. Therefore, the quantized system obtained from it, that coincides with $\left(\widehat{H}_{E}, \widehat{K}_{E_{1}}, \ldots, \widehat{K}_{E_{N-1}}\right)$, is quantum integrable. If the Robertson condition $\mathbf{C}_{K_{i}}=0, i=1, \ldots, N-1$, holds for the original system, it holds clearly for the system modified by the potentials $-\frac{\hbar^{2}}{2} E_{i}$, then the modified quantum system is also separable in $\left(q^{i}\right) .^{2}$ 


\section{EXAMPLES}

We show several detailed applications of the modified quantization. The following examples are inspired by the papers. ${ }^{16,17}$ The first two examples show how the freedom in the choice of the quantum correction for the Hamiltonian allows us to construct commuting symmetry operators: two geodesic Hamiltonians admitting a quadratic first integral considered in Ref. 17 are analysed. In Ref. 17, these examples were used to show how to apply the compatibility condition between the metric and the (conformal) Killing tensor. This condition means that a specific one-form, called obstruction, is closed

$$
d\left(\left(C_{s t}^{r a} \nabla_{r}-3 A_{s t}^{a}\right) K^{s t} g_{a b} d q^{b}\right)=0,
$$

where $K^{a b}$ are the contravariant components of the (conformal) Killing tensor associated with the first integral, while $\mathbf{A}$ and $\mathbf{C}$ are the Cotton-York tensor and the Weyl tensor, respectively. The scalar function $f$, local potential of the above closed one-form, has to be added to the standard conformally equivariant quantization of the quadratic first integral in order to ensure the commutation with the conformally invariant Laplacian. When the obstruction is not closed, the first integral is not associated with a second order symmetry of the conformally invariant Laplacian. Nevertheless, as shown in the examples below, a different scalar $E$ in the modified quantization could be used to create a different quantum Hamiltonian with a second-order symmetry operator.

\section{A. Example 1}

Let us consider the following three-dimensional Stäckel metric with an ignorable coordinate $q^{1}$,

$$
H=\frac{1}{2} p_{1}^{2}+\frac{1}{u\left(q^{2}\right)+v\left(q^{3}\right)}\left(\frac{1}{2} p_{2}^{2}+\frac{1}{2} p_{3}^{2}\right),
$$

and its quadratic first integral

$$
K=\frac{1}{u\left(q^{2}\right)+v\left(q^{3}\right)}\left(v\left(q^{3}\right) p_{2}^{2}-u\left(q^{2}\right) p_{3}^{2}\right) .
$$

Since the Ricci tensor is diagonal for all functions $u$ and $v$, by Remark 3, the ordinary Laplace-Beltrami quantization produces commuting quantum operators and no quantum correction is necessary, i.e., $E$ $=0$ and $E_{K}=0$ satisfy the compatibility condition (5).

On the contrary, since the metric is not conformally flat for arbitrary functions $u$ and $v$, we see that the quantum correction given by the scalar curvature,

$$
E=\mathrm{Sc}=\frac{1}{u\left(q^{2}\right)+v\left(q^{3}\right)}\left(\partial_{q^{2}}^{2}+\partial_{q^{3}}^{2}\right) \ln \left(u\left(q^{2}\right)+v\left(q^{3}\right)\right),
$$

is not compatible with $\mathbf{K}$. Indeed, the compatibility condition (6) reduces to $d \mathbf{K} d E=0$ which is satisfied if and only if

$$
\left(\partial_{q^{2}}^{2}+\partial_{q^{3}}^{2}\right) \partial_{q^{2}} \partial_{q^{3}} \ln \left(u\left(q^{2}\right)+v\left(q^{3}\right)\right)=0 .
$$

This analysis is in accordance with the results of Ref. 17 where it is shown that $\mathbf{K}$ is not associated with a symmetry of the conformally invariant Laplacian, when the above equation is not satisfied.

\section{B. Example 2}

Let us consider the following geodesic Hamiltonian:

$$
H=\frac{1}{2} p_{r}^{2}+\frac{1}{2} p_{z}^{2}+\frac{1}{2}\left(\frac{1}{r^{2}}-\frac{a^{2}}{z^{2}}\right) p_{\phi}^{2},
$$

obtained as a reduction of the Minkowski metric on $\mathbb{M}^{4}$ along the Killing vector $\mathbf{X}$ defined in pseudoCartesian coordinates $\left(x^{0}, x^{1}, x^{2}, x^{3}\right)$ as $\mathbf{X}=\left(x^{1} \partial_{0}+x^{0} \partial_{1}\right)+a\left(x^{1} \partial_{2}-x^{2} \partial_{1}\right)$, with $a \in \mathbb{R}-\{0\}$ (see Ref. 17 and the references therein). The metric of $H$ is conformal to the metric tensor of (13) with $u(r)=1 / r^{2}$ and $v(z)=-a / z^{2}$ and it is again a Stäckel metric. Indeed, a metric conformal to a Stäckel metric is of a Stäckel type if and only if the conformal factor is of the form $\sum_{i} f_{i}\left(q^{i}\right) g^{i i}$ (see Ref. 4). 
In this example, the conformal factor relating the two metrics is $g^{22}$. It is easy to check directly that the function,

$$
K=\frac{1}{2} p_{r}^{2}+\frac{1}{2 r^{2}} p_{\phi}^{2},
$$

is a quadratic first integral of $H$. The Ricci tensor of the metric of (15) is not diagonal in $(r, \phi, z)$ and the covariant form of $\delta \mathbf{C}_{K}$ is

$$
\left(\delta \mathbf{C}_{K}\right)^{b}=-\frac{3 a^{2}}{a^{2} r^{2}-z^{2}}\left(r\left(2 a^{2} r^{2}+3 z^{2}\right) d r+z\left(2 a^{2} z^{2}+3 r^{2}\right) d z\right)
$$

that is different from zero.

However, the quantum correction equation (5) can be solved for

$$
\begin{gathered}
E=-\frac{1}{8} \operatorname{Sc}-\frac{(a+1)^{2}}{4} \frac{a^{2} r^{2}+z^{2}}{a^{2} r^{2}-z^{2}}, \\
E_{K}=-\frac{a^{2}\left(a^{2} r^{2}+4 z^{2}\right)}{4\left(a^{2} r^{2}-z^{2}\right)^{2}},
\end{gathered}
$$

where

$$
\mathrm{Sc}=\frac{6 a^{2}\left(r^{2}+z^{2}\right)}{\left(a^{2} r^{2}-z^{2}\right)}
$$

is the scalar curvature of the metric tensor of (15). Also in this case, we recover the fact stated in Ref. 17 that $\mathbf{K}$ is not a symmetry of the conformally invariant Laplacian. Indeed, the quantum correction (17) requires an additional term to -Sc/8 (the correction which transforms a Laplacian in the conformally invariant Laplacian in a three-dimensional manifold) in order to satisfy the compatibility condition (6).

\section{Example 3}

In Ref. 16, the authors introduce a class of $N$-dimensional Hamiltonians, based upon the Tremblay-Turbiner-Winternitz system, that are superintegrable Stäckel systems, therefore admitting $N$ quadratic first integrals associated with the separation of variables, and $N-1$ other independent first integrals. These last constants of motion are polynomials in the momenta of degree depending on a rational parameter. These Hamiltonians are defined in non-conformally flat Riemannian manifolds for $N>3$; thus, their quantization requires quantum corrections in order to preserve the superintegrability of the corresponding quantum systems. In Ref. 16 is explicitly discussed the four-dimensional case and it is found that the quantum correction is a linear combination of the scalar curvature with an additional scalar determined from the Weyl tensor.

Here, we review the example of Ref. 16 step by step, under the assumption that also the other $N-1$ first integrals are quadratic in the momenta. Given the Hamiltonian

$$
\begin{array}{r}
H=L_{4}=p_{r}^{2}+\alpha r^{2}+\frac{L_{3}}{r^{2}}, \\
L_{3}=p_{\theta_{1}}^{2}+\frac{\beta_{1}}{\cos ^{2}\left(k_{1} \theta_{1}\right)}+\frac{L_{2}}{\sin ^{2}\left(k_{1} \theta_{1}\right)}, \\
L_{2}=p_{\theta_{2}}^{2}+\frac{\beta_{2}}{\cos ^{2}\left(k_{2} \theta_{2}\right)}+\frac{L_{1}}{\sin ^{2}\left(k_{2} \theta_{2}\right)}, \\
L_{1}=p_{\theta_{3}}^{2}+\frac{\beta_{3}}{\cos ^{2}\left(k_{3} \theta_{3}\right)}+\frac{\beta_{4}}{\sin ^{2}\left(k_{3} \theta_{3}\right)},
\end{array}
$$

we examine the classical and quantum second-order superintegrability of all $n$-dimensional subsystems $(n=2, \ldots, 4)$ with $\beta_{1}=\ldots=\beta_{4}=\alpha=0$ (we do not loose in generality since Equation (5) is unaffected by the addition of scalar potentials to the classical system) and determine the quantum corrections. 
In the following, we denote by $H_{i}$ the quadratic first integrals associated with the separation of variables, that are all in involution, and by $K_{i}$ the other quadratic first integrals.

The Hamiltonian (18) admits three additional independent second-degree first integrals for $k_{l}$ $=2^{l}, l=1, \ldots, 3$. Indeed, by setting $q^{4-j}=k_{j} \theta_{j}, p_{4-j}=p_{\theta_{j}} / k_{j}, j=1, \ldots, 3, q^{4}=r, p_{4}=p_{r}$, the Hamiltonian $H_{4}=H / 2$ becomes

$$
H_{4}=\frac{1}{2} p_{4}^{2}+\frac{4}{\left(q^{4}\right)^{2}}\left(\frac{1}{2} p_{3}^{2}+\frac{4}{\sin ^{2} q^{3}}\left(\frac{1}{2} p_{2}^{2}+\frac{4}{\sin ^{2} q^{2}}\left(\frac{1}{2} p_{1}^{2}\right)\right)\right) .
$$

It can be proved that all $H_{i}$ can be obtained recursively from $H_{1}=p_{1}^{2} / 2$ by applying the procedure of extension (see Ref. 10 and the references therein). Roughly speaking, this procedure transforms a Hamiltonian $L$ into a Hamiltonian of the form

$$
H=\frac{1}{2} p_{u}^{2}+\frac{m^{2}}{n^{2}} \alpha(u) L+f(u),
$$

with one more degree of freedom and admitting a polynomial first integral $K$ of degree depending on $\frac{m}{n}$ computable through a recursive procedure. For $\frac{m}{n}=2, K$ is quadratic (for more about the extension procedure, see Refs. 7-11). We leave for a next paper the details of the construction of suitable quantum corrections for a general extension with quadratic first integrals.

The two-dimensional system. The Hamiltonian,

$$
H_{2}=\frac{1}{2} p_{2}^{2}+\frac{4}{\sin ^{2} q^{2}}\left(\frac{1}{2} p_{1}^{2}\right)
$$

is defined on a two-dimensional constant curvature manifold and admits (together with $\mathrm{H}_{2}$ itself) the first integrals

$$
\begin{gathered}
H_{1}=\frac{1}{2} p_{1}^{2}, \\
K_{2}=\cos \left(q^{1}\right) p_{2}^{2}-4 \sin \left(q^{1}\right) \tan ^{-1}\left(q^{2}\right) p_{1} p_{2}-4 \cos \left(q^{1}\right) \tan ^{-2}\left(q^{2}\right) p_{1}^{2} .
\end{gathered}
$$

Both $H_{1}$ and $K_{2}$ are associated with commuting operators through the standard minimal quantization. Indeed, any constant curvature manifold is an Einstein manifold and therefore we have $\mathbf{C}_{K}=0$ for any symmetric Killing two-tensor $\mathbf{K}$.

The three-dimensional system. Let us consider the three-dimensional Hamiltonian

$$
H_{3}=\frac{1}{2} p_{3}^{2}+\frac{4}{\sin ^{2} q^{3}} H_{2},
$$

which is defined on a conformally flat manifold with a non-constant curvature

$$
\mathrm{Sc}_{3}=-6\left(1+\frac{1}{\sin ^{2} q^{3}}\right)
$$

with the Ricci tensor diagonal in these coordinates. Four additional first integrals are (24), (23), and (25) and

$$
K_{3}=\cos \left(q^{2}\right) p_{3}^{2}-4 \sin \left(q^{2}\right) \tan ^{-1}\left(q^{3}\right) p_{2} p_{3}-8 \cos \left(q^{2}\right) \tan ^{-2}\left(q^{3}\right) H_{2} .
$$

The Killing tensors $\mathbf{H}_{1}, \mathbf{H}_{2}$, and $\mathbf{K}_{2}$ associated with $H_{1}, H_{2}$, and $K_{2}$ satisfy the Carter condition and would not need quantum corrections, while the Killing tensor associated with $K_{3}$ does not satisfy the Carter condition and therefore a quantum correction is needed. Because of the conformal flatness, all the Killing tensors associated with the quadratic in the momenta first integrals satisfy Equation (6) of Proposition 2, with $E=-\frac{1}{8} \mathrm{Sc}_{3}$, in agreement with Ref. 17. Equation (5) is satisfied by the following quantum corrections for $\widehat{H}_{3}$ and $\widehat{K}_{3}$, respectively:

$$
\begin{gathered}
E_{3}=E\left(H_{3}, K_{3}\right)=-\frac{1}{8} \mathrm{Sc}_{3}=-\frac{3}{4}\left(1+\frac{1}{\sin ^{2} q^{3}}\right), \\
E_{K_{3}}=E\left(K_{3}, H_{3}\right)=-\frac{1}{2} \cos \left(q^{2}\right)\left(5 \tan ^{-2}\left(q^{3}\right)+2\right) .
\end{gathered}
$$


Now, in order to get simultaneous quantization for all first integrals, according to Theorem 6, we need to check that $E_{3}$ satisfies

$$
d \mathbf{H}_{1} d E_{3}=d \mathbf{H}_{2} d E_{3}=d \mathbf{K}_{2} d E_{3}=0,
$$

for the Killing tensors $\mathbf{H}_{1}, \mathbf{H}_{2}$, and $\mathbf{K}_{2}$. However, since $E_{3}$ depends on $q^{3}$ only and the first integrals of $H_{2}$ do not involve $p_{3}$, we have

$$
\mathbf{H}_{1} d E_{3}=\mathbf{H}_{2} d E_{3}=\mathbf{K}_{2} d E_{3}=0
$$

and, therefore, no quantum corrections are needed for $\widehat{H}_{2}, \widehat{H}_{1}$, and $\widehat{K}_{2}$. Thus, we get that

$$
\begin{gathered}
{\left[\widehat{H}_{3}-\frac{\hbar^{2}}{2} E_{3}, \widehat{K}_{3}-\frac{\hbar^{2}}{2} E_{K_{3}}\right]=\left[\widehat{H}_{3}-\frac{\hbar^{2}}{2} E_{3}, \widehat{K}_{2}\right]=0,} \\
{\left[\widehat{H}_{3}-\frac{\hbar^{2}}{2} E_{3}, \widehat{H}_{2}\right]=\left[\widehat{H}_{3}-\frac{\hbar^{2}}{2} E_{3}, \widehat{H}_{1}\right]=0 .}
\end{gathered}
$$

The four-dimensional system. The four-dimensional Hamiltonian (22) has the additional first integral,

$$
K_{4}=\cos \left(q^{3}\right) p_{4}^{2}-4 \sin \left(q^{3}\right)\left(q^{4}\right)^{-1} p_{3} p_{4}-8 \cos \left(q^{3}\right)\left(q^{4}\right)^{-2} H_{3},
$$

and the five constants of motion (23)-(27) inherited from the three-dimensional Hamiltonian. Its underlying manifold is not conformally flat, since the Weyl tensor does not vanish, but has a diagonalized Ricci tensor in $\left(q^{i}\right)$ (recall that this means that the Robertson condition is satisfied, hence $\left(q^{i}\right)$ are separable coordinates also for the Laplace-Beltrami operator). Following Ref. 16, we introduce the Weyl scalar

$$
W_{4}=\sqrt{3 W_{a b c d} W^{a b c d}}=\frac{24}{\left(q^{4}\right)^{2} \sin ^{2}\left(q^{3}\right)} .
$$

The quantum corrections for quantizing $H_{4}$ and $K_{4}$ (as we will show elsewhere in a more general context) are

$$
\begin{gathered}
E_{4}=E\left(H_{4}, K_{4}\right)=-\frac{1}{6} \operatorname{Sc}_{4}=\frac{4+3 \sin ^{2}\left(q^{3}\right)}{\left(q^{4}\right)^{2} \sin ^{2}\left(q^{3}\right)}, \\
E_{K_{4}}=E\left(K_{4}, H_{4}\right)=-2 \cos \left(q^{3}\right) \frac{4+3 \sin ^{2}\left(q^{3}\right)}{\left(q^{4}\right)^{2} \sin ^{2}\left(q^{3}\right)},
\end{gathered}
$$

while a pair of quantum corrections allowing simultaneous quantization for $H_{4}$ and $K_{3}$ is

$$
\begin{gathered}
\bar{E}_{4}=E\left(H_{4}, K_{3}\right)=\frac{3}{\left(q^{4}\right)^{2}}\left(1+\frac{1}{\sin ^{2} q^{3}}\right), \\
\bar{E}_{K_{3}}=E\left(K_{3}, H_{4}\right)=-\frac{1}{2} \cos \left(q^{2}\right)\left(5 \tan ^{-2}\left(q^{3}\right)+2\right) .
\end{gathered}
$$

We remark that $\bar{E}_{K_{3}}$ coincides with (29), while $\bar{E}_{4}$ is the product of (28) by $-4\left(q^{4}\right)^{-2}$, which is the coefficient of $\mathrm{H}_{3}$ in its extension $\mathrm{H}_{4}$. All other Killing tensors satisfy the Carter condition and do not need quantum correction. Hence, according to Theorem 5 , in order to get simultaneous modified quantization, we look for a function $E$ such that

$$
d \mathbf{K}_{4} d\left(E_{4}-E\right)=d \mathbf{K}_{3} d\left(\bar{E}_{4}-E\right)=0
$$

and

$$
d \mathbf{K}_{2} d E=0, \quad d \mathbf{H}_{i} d E=0, \quad(i=1, \ldots, 3) .
$$

By solving (31) for $E$ with the assumption that $E$ does not depend on $q^{1}$, we get

$$
E=\frac{3}{\left(q^{4}\right)^{2}}+\frac{3}{\left(q^{4}\right)^{2} \sin ^{2}\left(q^{3}\right)}+\frac{C_{1}+C_{2} \cos \left(q^{2}\right)}{\left(q^{4}\right)^{2} \sin ^{2}\left(q^{3}\right) \sin ^{2}\left(q^{2}\right)}+C_{3}\left(q^{4}\right)^{2}+C_{4},
$$


where $C_{1}, \ldots, C_{4}$ are arbitrary constants. We observe that for $C_{i}=0$ we get the quantum correction used in Ref. 16 which is a linear combination of the scalar curvature and of the Weyl scalar. By imposing the further condition $d \mathbf{K}_{2} d E=0$, we get

$$
\left(\cos ^{4}\left(q^{2}\right)-6 \cos ^{2}\left(q^{2}\right)-3\right) C_{2}-8 C_{1} \cos \left(q^{2}\right)=0 .
$$

Thus, we need $C_{1}=C_{2}=0$. Furthermore, for this choice of the constants $C_{i}$, we have $\mathbf{H}_{1} d E=\mathbf{H}_{2} d E$ $=\mathbf{K}_{2} d E=0$ and $d \mathbf{H}_{3} d E=0$. Hence, the required quantum correction for $\widehat{H}_{4}$ is

$$
E=\frac{3}{\left(q^{4}\right)^{2}}+\frac{3}{\left(q^{4}\right)^{2} \sin ^{2}\left(q^{3}\right)}+C_{3}\left(q^{4}\right)^{2}+C_{4} .
$$

The modified Hamiltonian operator $\left(C_{4} \in \mathbb{R}\right)$,

$$
\widehat{H}_{4}-\frac{\hbar^{2}}{2}\left(3 \frac{1+\sin ^{2} q^{3}}{\left(q^{4}\right)^{2} \sin ^{2} q^{3}}+C_{4}\left(q^{4}\right)^{2}\right),
$$

commute with the six following modified operators:

$$
\begin{aligned}
& \widehat{K}_{4}-\hbar^{2} \cos \left(q^{3}\right)\left(C_{4}\left(q^{4}\right)^{2}-3 \frac{1+\sin ^{2} q^{3}}{\left(q^{4}\right)^{2} \sin ^{2} q^{3}}\right), \\
& \widehat{K}_{3}+\frac{\hbar^{2}}{4} \cos \left(q^{2}\right)\left(5 \tan ^{-2}\left(q^{3}\right)+2\right), \\
& \widehat{K}_{2}, \\
& \widehat{H}_{3}-\frac{3 \hbar^{2}}{8 \sin ^{2} q^{3}}, \\
& \widehat{H}_{2}, \\
& \widehat{H}_{1} .
\end{aligned}
$$

Remark 4. Operators (34)-(38) are the five symmetry operators of (36) determined in the threedimensional case.

The 5-dimensional system. As a further step, we consider a Hamiltonian $H_{5}$ with five degrees of freedom. In this case, to apply the extension procedure, the four dimensional Hamiltonian (22) must be replaced by

$$
H^{\prime}{ }_{4}=\frac{1}{2} p_{4}^{2}+\frac{4}{\sin ^{2} q^{4}}\left(\frac{1}{2} p_{3}^{2}+\frac{4}{\sin ^{2} q^{3}}\left(\frac{1}{2} p_{2}^{2}+\frac{4}{\sin ^{2} q^{2}}\left(\frac{1}{2} p_{1}^{2}\right)\right)\right)
$$

that admits the first integrals

$$
K^{\prime}{ }_{4}=\cos \left(q^{3}\right) p_{4}^{2}-4 \sin \left(q^{3}\right) \tan ^{-1}\left(q^{4}\right) p_{3} p_{4}-8 \cos \left(q^{3}\right) \tan ^{-2}\left(q^{4}\right) H_{3},
$$

and the five constants of motion (23)-(27) inherited from the three-dimensional case. The underlying manifold of $\mathrm{H}_{4}^{\prime}$ is not conformally flat, since the Weyl tensor is not zero, but has the Ricci tensor diagonalized in $\left(q^{i}\right)$. The Weyl scalar is

$$
W^{\prime}{ }_{4}=\sqrt{3 W^{\prime}{ }_{a b c d} W^{\prime a b c d}}=\frac{24}{\sin ^{2}\left(q^{4}\right) \sin ^{2}\left(q^{3}\right)} .
$$

The quantum corrections for quantizing $\mathrm{H}_{4}^{\prime}$ and $\mathrm{K}_{4}{ }_{4}$ (as we will show elsewhere in a more general context) are

$$
\begin{gathered}
E^{\prime}{ }_{4}=E\left({H^{\prime}}_{4}, K^{\prime}{ }_{4}\right)=-\frac{1}{6} \mathrm{Sc}_{4}=\frac{4+3 \sin ^{2} q^{3}}{\sin ^{2}\left(q^{4}\right) \sin ^{2}\left(q^{3}\right)}+2, \\
E_{K_{4}}^{\prime}=E\left(K^{\prime}{ }_{4}, H^{\prime}{ }_{4}\right)=-2 \cos \left(q^{3}\right)\left(\frac{4+3 \sin ^{2} q^{3}}{\tan ^{2}\left(q^{4}\right) \sin ^{2}\left(q^{3}\right)}+1\right),
\end{gathered}
$$


while, a pair of quantum corrections allowing simultaneous quantization for $H^{\prime}{ }_{4}$ and $K_{3}$ is

$$
\begin{gathered}
\bar{E}_{4}^{\prime}{ }_{4}=E\left({H^{\prime}}_{4}, K_{3}\right)=\frac{3}{\sin ^{2} q^{4}}\left(1+\frac{1}{\sin ^{2} q^{3}}\right), \\
\bar{E}_{K_{3}}=E\left(K_{3}, H^{\prime}{ }_{4}\right)=E\left(K_{3}, H_{3}\right)=-\frac{1}{2} \cos \left(q^{2}\right)\left(5 \tan ^{-2}\left(q^{3}\right)+2\right) .
\end{gathered}
$$

By repeating similar computations of above, we find that a quantum correction allowing the simultaneous quantization of ${\mathrm{H}^{\prime}}_{4}$ with all the other first integrals is given by

$$
E^{\prime}=\frac{3}{\sin ^{2} q^{4}}+\frac{3}{\sin ^{2}\left(q^{4}\right) \sin ^{2}\left(q^{3}\right)}+C_{4} \tan ^{2} q^{4},
$$

with $C_{4} \in \mathbb{R}$. The modified Hamiltonian operator,

$$
{\widehat{H^{\prime}}}_{4}-\frac{\hbar^{2}}{2}\left(\frac{3}{\sin ^{2} q^{4}}\left(1+\frac{1}{\sin ^{2} q^{3}}\right)+C_{4} \tan ^{2} q^{4}\right)
$$

commute with the modified operator

$$
{\widehat{K^{\prime}}}_{4}-\hbar^{2} \cos \left(q^{3}\right)\left(C_{4} \tan ^{-2}\left(q^{4}\right)-\tan ^{2}\left(q^{4}\right)-3-\frac{3}{\sin ^{2} q^{3}}\right),
$$

and with the four operators (34)-(38).

The five dimensional extended Hamiltonian is therefore

$$
H_{5}=\frac{1}{2} p_{5}^{2}+\frac{4}{\left(q^{5}\right)^{2}}\left(\frac{1}{2} p_{4}^{2}+\frac{4}{\sin ^{2} q^{4}}\left(\frac{1}{2} p_{3}^{2}+\frac{4}{\sin ^{2} q^{3}}\left(\frac{1}{2} p_{2}^{2}+\frac{4}{\sin ^{2} q^{2}}\left(\frac{1}{2} p_{1}^{2}\right)\right)\right)\right),
$$

with first integrals

$$
K_{5}=\cos \left(q^{4}\right) p_{5}^{2}-4 \sin \left(q^{4}\right)\left(q^{5}\right)^{-1} p_{4} p_{5}-8 \cos \left(q^{4}\right)\left(q^{5}\right)^{-2} H^{\prime}{ }_{4},
$$

and the seven constants of motion of ${H^{\prime}}_{4}$. The underlying manifold has the scalar curvature and the Weyl scalar given by

$$
\begin{gathered}
\operatorname{Sc}_{5}=-12 \frac{6 \sin ^{2}\left(q^{3}\right)+8+3 \sin ^{2}\left(q^{4}\right) \sin ^{2}\left(q^{3}\right)}{\left(q^{5}\right)^{2} \sin ^{2}\left(q^{4}\right) \sin ^{2}\left(q^{3}\right)}, \\
W_{5}=4 \sqrt{6} \frac{\sqrt{3 \sin ^{4}\left(q^{3}\right)+8 \sin ^{2}\left(q^{3}\right)+48}}{\left(q^{5}\right)^{2} \sin ^{2}\left(q^{4}\right) \sin ^{2}\left(q^{3}\right)} .
\end{gathered}
$$

We can again find a quantum correction for all second order operators and a simultaneous quantum correction

$$
E=C_{5}\left(q^{5}\right)^{2}+\frac{27}{4\left(q^{5}\right)^{2}}+\frac{12}{\left(q^{5}\right)^{2} \sin ^{2} q^{4}}\left(1+\frac{1}{\sin ^{2} q^{3}}\right),
$$

with $C_{5} \in \mathbb{R}$, such that $\widehat{H}_{5}-\frac{\hbar^{2}}{2} E$ commutes with the modified operator

$$
\widehat{K}_{5}-\hbar^{2} \cos \left(q^{4}\right)\left(C_{5}\left(q^{5}\right)^{2}-\frac{21}{4\left(q^{5}\right)^{2}}-\frac{12}{\left(q^{5}\right)^{2} \sin ^{2} q^{4}}\left(1+\frac{1}{\sin ^{2} q^{3}}\right)\right),
$$

with the operators (41) and (42) for $C_{4}=0$ and with the four operators (34)-(38).

Remark 5. In dimension 4, the simultaneous quantum correction for $C_{4}=0$ is a linear combination of $\mathrm{Sc}_{4}$ and $W_{4}$ (see also Ref. 16), while in dimension 5 any simultaneous quantum correction (46) of $H^{\prime}{ }_{4}$ with all the other first integrals is not a rational function of $\mathrm{Sc}_{5}$ and $W_{5}$.

Remark 6. The construction of above can be iterated until dimension $N$, for $i<N$

$$
H_{i}=\frac{1}{2} p_{i}^{2}+\frac{4}{\sin ^{2}\left(q^{i}\right)} H_{i-1}
$$




$$
K_{i}=\cos \left(q^{i-1}\right) p_{i}^{2}-4 \sin \left(q^{i-1}\right) \tan ^{-1}\left(q^{i}\right) p_{i} p_{i-1}-8 \cos \left(q^{i-1}\right) \tan ^{-2}\left(q^{i}\right) H_{i-1},
$$

and for $i=N$

$$
\begin{gathered}
H_{N}=\frac{1}{2} p_{N}^{2}+\frac{4}{\left(q^{N}\right)^{2}} H_{N-1} \\
K_{N}=\cos \left(q^{N-1}\right) p_{N}^{2}-4 \sin \left(q^{N-1}\right)\left(q^{N}\right)^{-1} p_{N} p_{N-1}-8 \cos \left(q^{N-1}\right)\left(q^{N}\right)^{-2} H_{N-1} .
\end{gathered}
$$

It is easy to see that in these coordinates the metric tensor of $H_{N}$ has a diagonal Ricci tensor; thus the $\widehat{H}_{i}$ do not need quantum corrections. We leave for a further paper a more detailed discussion of the general case.

\section{CONCLUSIONS}

By Theorems 1, 5, and 6, we characterize those natural Hamiltonian systems with quadratic in the momenta constants of motion that are preserved as dynamical constants after the Laplace-Beltrami quantization, up to the addition of scalar potentials. The examples illustrate both the generality and the usefulness of our approach, not restricted to any a priori assumption of the Hamiltonian quantum correction. In Example 3, we consider a family of Hamiltonians, arising from the procedure of "extension" of lower-dimensional natural Hamiltonians, admitting a quadratic in the momenta constant of motion generated by the extension procedure. In a future article, we will study the LB quantization of generic extended Hamiltonians with a similar quadratic constant of motion. Some of the results of this study are already introduced here in Example 3. We remark that any extended Hamiltonian depends on some freely chosen rational number $k$ and admits a constant of motion, polynomial in the momenta, of degree determined by $k$. Therefore, extended Hamiltonians represent an excellent source of examples for any quantization theory, not restricted only to polynomials quadratic in the momenta. See, for example, Ref. 18.

\section{ACKNOWLEDGMENTS}

The authors are grateful to Jonathan Kress for suggesting to apply modified quantization to example 2 and to Francisco Herranz for introducing us to the theory of modified LB quantization.

${ }^{1}$ Ballesteros, A., Enciso, A., Herranz, F. J., Ragnisco, O., and Riglioni, D., "Quantum mechanics on spaces of nonconstant curvature: The oscillator problem and superintegrability”, Ann. Phys. 326(8), 2053-2073 (2011).

${ }^{2}$ Benenti, S., Chanu, C., and Rastelli, G., "Remarks on the connection between the additive separation of the Hamilton-Jacobi equation and the multiplicative separation of the Schrödinger equation. I. The completeness and Robertson conditions,' J. Math. Phys. 43, 5183-5222 (2002).

${ }^{3}$ Benenti, S., Chanu, C., and Rastelli, G., "Remarks on the connection between the additive separation of the Hamilton-Jacobi equation and the multiplicative separation of the Schrödinger equation. II. First integrals and symmetry operators," J. Math. Phys. 43, 5223-5253 (2002).

${ }^{4}$ Benenti, S., Chanu, C., and Rastelli, G., "Variable-separation theory for the null Hamilton-Jacobi equation," J. Math. Phys. 46, 042901 (2005).

${ }^{5}$ Błaszak, M., Marciniak, K., and Domanski, Z., “Separable quantizations of Stäckel systems,” Ann. Phys. 371, 460-477 (2016).

${ }^{6}$ Błaszak, M. and Marciniak, K., "Classical and quantum superintegrabilty of Stäckel systems," SIGMA 13, 008 (2017).

${ }^{7}$ Chanu, C., Degiovanni, L., and Rastelli, G., "First integrals of extended Hamiltonians in (n+1)-dimensions generated by powers of an operator," SIGMA 7(038), 12 (2011).

${ }^{8}$ Chanu, C., Degiovanni, L., and Rastelli, G., "Generalizations of a method for constructing first integrals of a class of natural Hamiltonians and some remarks about quantization,” J. Phys.: Conf. Ser. 343, 012101 (2011); e-print arXiv:1111.0030.

${ }^{9}$ Chanu, C., Degiovanni, L., and Rastelli, G., "Extensions of Hamiltonian systems dependent on a rational parameter," J. Math. Phys. 55, 122703 (2014).

${ }^{10}$ Chanu, C., Degiovanni, L., and Rastelli, G., “The Tremblay-Turbiner-Winternitz system as extended Hamiltonian,” J. Math. Phys. 55, 122701 (2014).

${ }^{11}$ Chanu, C., Degiovanni, L., and Rastell, G., "Superintegrable extensions of superintegrable systems," SIGMA 8(070), 12 (2012).

12 Carter, B., "Killing tensor quantum numbers and conserved currents in curved space," Phys. Rev. D 16, 3395 (1977).

${ }^{13}$ Duval, C., Lecomte, P., and Ovsienko, V., "Conformally invariant quantization," Ann. Inst. Fourier 49, 1999-2029 (1999).

${ }^{14}$ Duval, C. and Ovsienko, V., “Conformally equivariant quantum Hamiltonians,” Sel. Math. 7, 291-320 (2001). 
${ }^{15}$ Duval, C. and Valent, G., “A new integrable system on the sphere and conformally equivariant quantization,” J. Geom. Phys. 61(8), 1329-1347 (2011).

${ }^{16}$ Kalnins, E. G., Kress, J. M., and Miller, W., Jr., "Superintegrability in a non-conformally-flat space,” J. Phys. A: Math. Theor. 46(2), 022002 (2012).

${ }^{17}$ Michel, J.-P., Radoux, F., and Šilhan, J., "Second order symmetries the conformal Laplacian," SIGMA 10(016), 26 (2014).

${ }^{18}$ Rastelli, G., "Born-Jordan and Weyl quantizations of the 2D anisotropic harmonic oscillator," SIGMA 12(081), 7 (2016). 